\title{
Decision-Making Algorithm for Multisensor Fusion Based on Grey Relation and DS Evidence Theory
}

\author{
Fang Ye, Jie Chen, Yibing Li, and Jian Kang \\ College of Information and Communication Engineering, Harbin Engineering University, Harbin 150001, China \\ Correspondence should be addressed to Yibing Li; liyibing0920@sina.cn
}

Received 12 May 2016; Accepted 22 September 2016

Academic Editor: Biswajeet Pradhan

Copyright ( 2016 Fang Ye et al. This is an open access article distributed under the Creative Commons Attribution License, which permits unrestricted use, distribution, and reproduction in any medium, provided the original work is properly cited.

\begin{abstract}
Decision-making algorithm, as the key technology for uncertain data fusion, is the core to obtain reasonable multisensor information fusion results. DS evidence theory is a typical and widely applicable decision-making method. However, DS evidence theory makes decisions without considering the sensors' difference, which may lead to illogical results. In this paper, we present a novel decision-making algorithm for uncertain fusion based on grey relation and DS evidence theory. The proposed algorithm comprehensively takes consideration of sensor's credibility and evidence's overall discriminability, which can solve the uncertainty problems caused by inconsistence of sensors themselves and complexity of monitoring environment and simultaneously ensure the validity and accuracy of fusion results. The innovative decision-making algorithm firstly obtains the sensor's credibility through the introduction of grey relation theory and then defines two impact factors as sensor's credibility and evidence's overall discriminability according to the focal element analyses and evidence's distance analysis, respectively; after that, it uses the impact factors to modify the evidences and finally gets more reasonable and effective results through DS combination rule. Simulation results and analyses demonstrate that the proposed algorithm can overcome the trouble caused by large evidence conflict and one-vote veto, which indicates that it can improve the ability of target judgment and enhance precision of uncertain data fusion. Thus the novel decisionmaking method has a certain application value.
\end{abstract}

\section{Introduction}

In practical applications, single sensor is difficult to meet the requirements like target accuracy and identification performance. Thus, there is a broad application of decisionmaking algorithm on data fusion about target's attributes, characteristics, and types through comprehensive processing of information obtained from multisensor. Currently, data decision-making technology [1-3] based on multisensor is highly valued by scholars at home and abroad. In addition, a lot of theorems and algorithms emerge in the area of data decision-making. However, due to constraints on the attributes as well as the types of data, there is still no unified theoretical framework or unique algorithm for classification issue of multisensor data decision-making.

For multisensor decision-making field, the traditional algorithms are statistical method [4], empirical reasoning [5], voting method [6], Bayesian inference [7], template method [5], and adaptive neural network [8], among others. These typical methods all can settle the decision fusion of multisensor information to some extent, whereas they all have some defects. Statistical method, empirical reasoning, and voting method are too simple to achieve the reliable decision results for multisensor information fusion. Bayesian inference needs the prior knowledge of environment to finish the reasoning, which cannot be guaranteed in actual applications. And template method would waste time and energy of system when selecting the suitable template according to certain rules. Although adaptive neural network can fulfill a reasonable decision fusion, it is usually not adopted in practical applications because of its large computation complexity. DS evidence theory $[9,10]$ is favored for its ability of dealing with uncertainty, integration of measurement information, and reasonable theoretical derivation. Thus, DS evidence theory has become the mainstream method in multisensor decisionmaking field.

As a wildly used decision-making algorithm for uncertain data fusion, DS evidence theory is able to deal with the uncertainty and imprecision of multisensor information fusion. 
Hence, DS evidence theory can properly handle the inconsistency of sensor conditions and complexity of monitoring environment. With its introduction and perfection put forward by Dempster and Shafer, respectively, DS evidence theory occupies a lot in the development of intelligent computing and identification theory for multisensor information fusion. Along with its development, DS evidence theory has been widely applied in various fields, like pattern recognition [11], target identification [12], cognitive radio network [13], fault diagnosis [14], signal recognition [15], and decision-making [16], among others. Although there are some problems of DS evidence theory itself, these problems can be effectively solved through rigorous theoretical derivation, scientific improvements, and combination with other methods. For example, a new entropy, named as Deng entropy, is proposed in [17] to handle the uncertain measure of BPA, which is the generalization of Shannon entropy. The new entropy provides a promising way to measure the uncertainty of multisensor fusion system. Besides, Deng entropy is applied in [18] to realize the measurement of information volume of the evidence. This improvement makes the application of DS evidence theory with more validity and robustness. Due to limit space, the classic modified methods [19-31] are exhibited in references and partially taken as compared methods in Section 5.2.

In this paper, systematic research is implemented on DS evidence theory, and the multisensor decision-making algorithm is realized by the combination of DS evidence theory and grey relation analysis [32, 33]. The proposed decision-making algorithm for uncertain data fusion firstly utilizes sensors' report generator to settle the acquisition processing of sensor's credibility by the introduction of grey relation theory. Then, the sensor's credibility is consecutively adjusted by two different processes of consistency and conflict analysis in focal elements. At the same time, the novel method defines the evidence's overall discriminability according to the concept of evidence's distance function. Finally, the original evidences are modified by two impact factors as sensor's credibility and evidence's overall discriminability, which can ensure getting more reasonable and effective decision-making results after evidences combine.

This paper is organized as follows. The theoretical theorem and derivation of DS evidence theory and grey relation theory are briefly introduced in the next section. And the implementation diagram and flow chart of uncertain data fusion system are given in Section 3. Then, Section 4 highlights the implementation method and specific steps of the new decision-making algorithm for uncertain data fusion, and Section 5 presents the simulation results and comparative analyses. Concluding remarks are given in the last section of this paper.

\section{Theoretical Foundations}

DS evidence theory and grey relation theory are separately presented in this section, which are the foundations of the novel decision-making algorithm in this paper.
2.1. DS Evidence Theory. DS evidence theory, also called Dempster-Shafer theory, is an effective data decision-making method to deal with the uncertainty of multisensor information fusion system. Relative to probability theory [5], DS evidence theory can settle imprecise data and has a more extensive application area. Similar to Bayesian inference [7], DS evidence theory uses the prior probability to represent the evidence interval of posterior probability, which can quantify the credible degree and plausibility degree of propositions. DS evidence theory is briefly comprised by the following four key points.

2.1.1. Frame of Discernment and the Power Set. In DS model, the frame of discernment (FoD) denoted by $\Theta$ indicates a set of $N$ mutually exclusive and exhaustive hypotheses, which represents all interested propositions. And FoD is defined as the form of function set as

$$
\Theta=\left\{H_{1}, H_{2}, \ldots, H_{N}\right\}=\left\{H_{i} \mid i=1,2, \ldots, N\right\},
$$

where $H_{i}$ is the $i$ th hypothesis belonging to $\Theta$ and $N$ is the number of hypotheses.

On the basis of FoD, we can derive $2^{\Theta}$ as the power set, which is composed of $2^{N}$ propositions of $\Theta$ (all subsets of FoD).

$$
\begin{aligned}
2^{\Theta} & =\left\{\emptyset,\left\{H_{1}\right\},\left\{H_{2}\right\}, \ldots,\left\{H_{N}\right\},\left\{H_{1}, H_{2}\right\},\left\{H_{1}, H_{3}\right\}, \ldots,\right. \\
& \left.\left\{H_{1}, H_{N}\right\}, \ldots,\left\{H_{1}, H_{2}, \ldots, H_{N}\right\}\right\},
\end{aligned}
$$

where $\emptyset$ is the empty set, which belongs to any propositions.

2.1.2. Basic Probability Assignment. The basic probability assignment (BPA) is a mass function $m: 2^{\Theta} \rightarrow[0,1]$ defined on $2^{\Theta}$, which should satisfy the following demands:

$$
\begin{aligned}
m(\emptyset) & =0, \\
\sum_{A \subseteq \Theta} m(A) & =1,
\end{aligned}
$$

$\forall A \in 2^{\Theta} \cdot m(A)$ is called the mass function of proposition $A$ that represents the basic belief degree and initial support degree strictly assigned to proposition $A$ [17].

Due to the lack of further knowledge, $m(A)$ cannot be subdivided. Any proposition satisfying that $m(A)>0(A \in$ $\left.2^{\Theta}\right)$ is called the focal element, and the set of all focal elements is named as the core of BPA.

2.1.3. Belief Function and Plausibility Function. DS evidence theory designates two uncertain measurements as the belief function (Bel) and plausibility function (Pl). Similar to the definition of BPA, Bel and Pl can be defined, respectively, as

$$
\begin{aligned}
\operatorname{Bel}(A) & =\sum_{B \subseteq A} m(B), \\
\operatorname{Pl}(A) & =\sum_{B \cap A \neq \emptyset} m(B),
\end{aligned}
$$




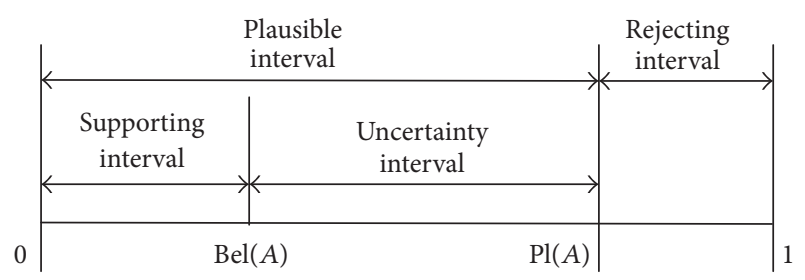

Figure 1: Relationship diagram of $\operatorname{Bel}(A)$ and $\operatorname{Pl}(A)$.

$\forall A \in 2^{\Theta}$, where $\operatorname{Bel}(A)$ is interpreted as the low probability of $A$, while $\operatorname{Pl}(A)$ is interpreted as the upper probability of $A$. The relationship between $\operatorname{Bel}(A)$ and $\operatorname{Pl}(A)$ is derived as follows:

$$
\begin{aligned}
\operatorname{Bel}(A) & \leq \operatorname{Pl}(A), \\
\operatorname{Pl}(A) & =1-\operatorname{Bel}(\bar{A}),
\end{aligned}
$$

where $\bar{A}$ is the complement set of $A$.

According to the relationship between $\operatorname{Bel}(A)$ and $\operatorname{Pl}(A)$, DS evidence theory also divides the evidence interval into supporting interval, uncertainty interval, and rejecting interval, which are shown in Figure 1.

The interval $[\operatorname{Bel}(A), \operatorname{Pl}(A)]$ is named the uncertainty interval, which represents the uncertainty and imprecision of multisensor fusion system.

The concept of uncertainty interval is similar to probability, but not entirely expressed as probability. The interval makes the proposition possibly real; that is, it does not directly support or reject the proposition. That feature demonstrates that DS evidence theory needs weaker axiom than probability theory and can represent the difference between uncertainty and unknown of proposition [9]. Thus, DS evidence theory is the generalization of probability theory and is an effective solution method when the prior knowledge is absent.

2.1.4. DS Combination Rule. DS evidence theory provides a useful evidence combination function. Suppose that there are 2 independent and not completely conflict evidences that exist on the same FoD in system; we can get a synthesis support degree for propositions by DS combination rule. The combination rule can be computed by the orthogonal sum of their mass functions; that is,

$$
\begin{aligned}
m(A) & =\left[m_{1} \oplus m_{2}\right](A) \\
& =\frac{1}{1-k} \sum_{A_{i} \cap B_{j}=A} m_{1}\left(A_{i}\right) \cdot m_{2}\left(B_{j}\right),
\end{aligned}
$$

$\forall A \in 2^{\Theta}$, where $\oplus$ represents the orthogonal sum operator. $k$ is the global conflict factor, which demonstrates the conflict degree between $m_{1}$ and $m_{2}$ :

$$
k=1-\sum_{A_{i} \cap B_{j}=\emptyset} m_{1}\left(A_{i}\right) \cdot m_{2}\left(B_{j}\right) .
$$

If $k$ is close to 0,2 evidences are on the verge of conformity. While $k$ is close to 1,2 evidences are totally conflict. The denominator $1 /(1-k)$ is the normalization factor which ensures that (3) are contented.

The equations and properties of DS combination rules based on 2 evidences are exhibited here; readers can deduce the equations and properties of multiple evidences' synthesis with similar principle.

Obviously, the DS combination rule satisfies both commutative law and associate law.

$$
\begin{aligned}
m_{1} \oplus m_{2} & =m_{2} \oplus m_{1}, \\
\left(m_{1} \oplus m_{2}\right) \oplus m_{3} & =m_{1} \oplus\left(m_{2} \oplus m_{3}\right) .
\end{aligned}
$$

2.2. Grey Relation Theory. Grey relation theory [34] is the quantity processing and ordering procedure of systems with incomplete information or uncertain data. It can be seen as a global analysis of system. Since appropriate reference is essential to obtain reasonable sensor credibility result, a certain sensor is used as a comparative standard to determine the credibility degree of multisensor [35].

2.2.1. Grey Relation Factor. Grey relation factor is the basis of grey relation analysis [32]. The space of Grey relation factors is determined by sequence that has properties as comparability, accessibility, and extreme consistency.

Suppose that the sequences of system are $x_{i}=\left[x_{i}(1)\right.$, $\left.x_{i}(2), \ldots, x_{i}(n)\right], i=0,1,2, \ldots, m$, where $x_{0}$ is the reference sequence and $x_{i}, i=1,2, \ldots, m$, is the comparison sequence. $\gamma\left(x_{0}(k), x_{i}(k)\right)$ represents the comparison measurement of $x_{0}$ and $x_{i}$ at the $k$ th point in grey relation factors' space. Then we define the grey relation factor of $x_{i}$ as $\gamma\left(x_{0}, x_{i}\right)$, which is the average value of $\gamma\left(x_{0}(k), x_{i}(k)\right)$ at all points. Hence, the degree of grey relation factor is defined as

$$
\gamma\left(x_{i}, x_{0}\right)=\frac{1}{n} \sum_{k=1}^{n} \gamma\left(x_{0}(k), x_{i}(k)\right)
$$

where the comparison measurement of $x_{0}$ and $x_{i}$ is expressed as

$$
\begin{aligned}
& \gamma\left(x_{0}(k), x_{i}(k)\right) \\
& \quad=\frac{\min _{i} \min _{k} \Delta_{0 i}(k)+\zeta \max _{i} \max _{k} \Delta_{0 i}(k)}{\Delta_{0 i}(k)+\zeta \max _{i} \max _{k} \Delta_{0 i}(k)},
\end{aligned}
$$

where $\zeta \in[0,1]$ is the resolution index and $\Delta_{0 i}(k)$ is the discriminative information.

2.2.2. Properties of Grey Relation Factor. It is apparent that the grey relation factor has the following elementary properties [34]:

(1) Normativity:

$$
\begin{aligned}
0 & \leq \gamma\left(x_{0}, x_{i}\right) \leq 1, \\
\gamma\left(x_{0}, x_{i}\right) & =1 \Longleftrightarrow \\
x_{0} & =x_{i},
\end{aligned}
$$




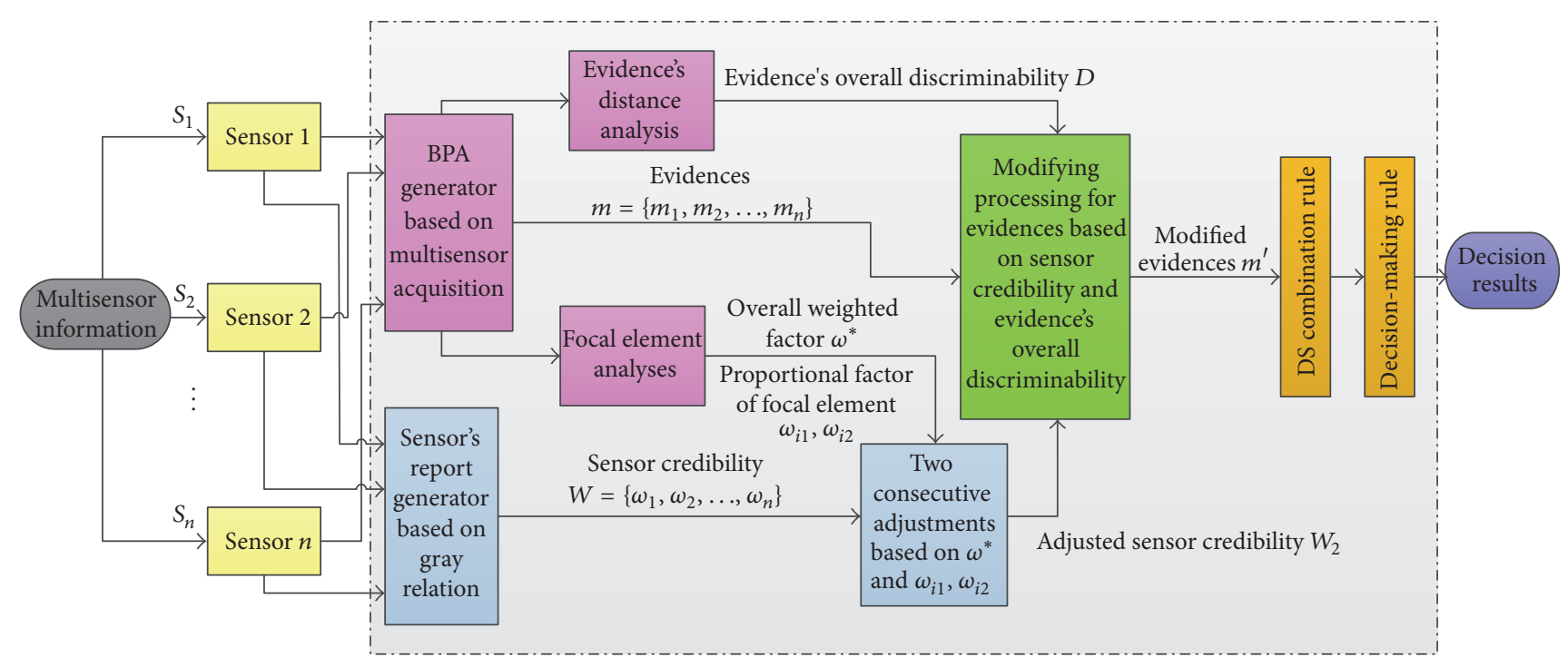

FIGURE 2: Implementation diagram of uncertain data fusion system.

$$
\begin{gathered}
\gamma\left(x_{0}, x_{i}\right)=0 \Longleftrightarrow \\
x_{0}, x_{i} \in \emptyset .
\end{gathered}
$$

(2) Symmetry:

$$
\gamma\left(x_{0}, x_{i}\right)=\gamma\left(x_{i}, x_{0}\right)
$$

(3) Accessibility:

$$
\Delta_{0 i}(k) \downarrow=\gamma\left(x_{0}(k), x_{i}(k)\right) \uparrow .
$$

Namely, the smaller the discriminative information $\Delta_{0 i}(k)$ is, the bigger the comparison measurement $\gamma\left(x_{0}(k), x_{i}(k)\right)$ is.

\section{The Implementation Diagram of Uncertain Data Fusion System}

According to the proposed decision-making algorithm, the implementation diagram of uncertain data fusion system is defined in Figure 2.

The structure of the proposed decision-making algorithm is marked by the rectangular block with imaginary lines in Figure 2. It is evident that the new decision-making method is comprised of four parts. Thus, we can get the flow chart in Figure 3.

The new method is realized by the following four steps.

Step 1. Obtain sensor's credibility through sensors' report generator based on grey relation theory and consecutively adjust sensor's credibility, respectively, through overall weighted factor analysis and proportional factor analysis. Then, filtrate the evidences according to sensor's credibility's value.

Step 2. Define evidence's overall discriminability by evidences' distance analysis.
Step 3. Modify the original evidences by two impact factors as sensor's credibility and evidence's overall discriminability.

Step 4. Combine the modified evidences by proper DS combination rule, and put the synthetic results into decisionmaking rule to get the final decision results.

\section{The New Decision-Making Method Based on Grey Relation and DS Evidence Theory}

As described last section, the particular procedures of the new method are presented. The novel decision-making algorithm takes two impact factors as sensor's credibility and evidence's overall discriminability to modify the original evidences, respectively, by focal element analyses and evidences' distance analysis. The proposed algorithm can settle system's uncertainty caused by inconsistency of sensor conditions and complexity of monitoring environment. Therefore, the new method is able to guarantee the decision accuracy of data fusion.

\subsection{Two Consecutive Adjustments of Sensor's Credibility}

4.1.1. Generation of Sensor's Credibility Based on Grey Relation. In this part, the concept of grey relation theory is utilized to analyze sensor's credibility by generating sensor's report.

For multisensor information fusion system, let us denote the exclusive and exhaustive FoD as $\Theta=\left\{H_{1}, H_{2}, \ldots, H_{m}\right\}$, where $m$ is the number of hypotheses. Taking a sensor as template, we can associate the measurement information provided by each sensor with the template sensor. Then sensor's credibility report is built.

Suppose $\mathbf{X}_{0}=\left\{\mathbf{X}_{0}(j) \mid j=1,2, \ldots, M\right\}$ is the measurement information of the reference sensor, $\mathbf{X}_{i}=\left\{\mathbf{X}_{i}(j) \mid j=\right.$ $1,2, \ldots, M\}$ is the measurement information of multisensor, where the index $i=1,2, \ldots, n$ represents the $i$ th sensor, $n$ 


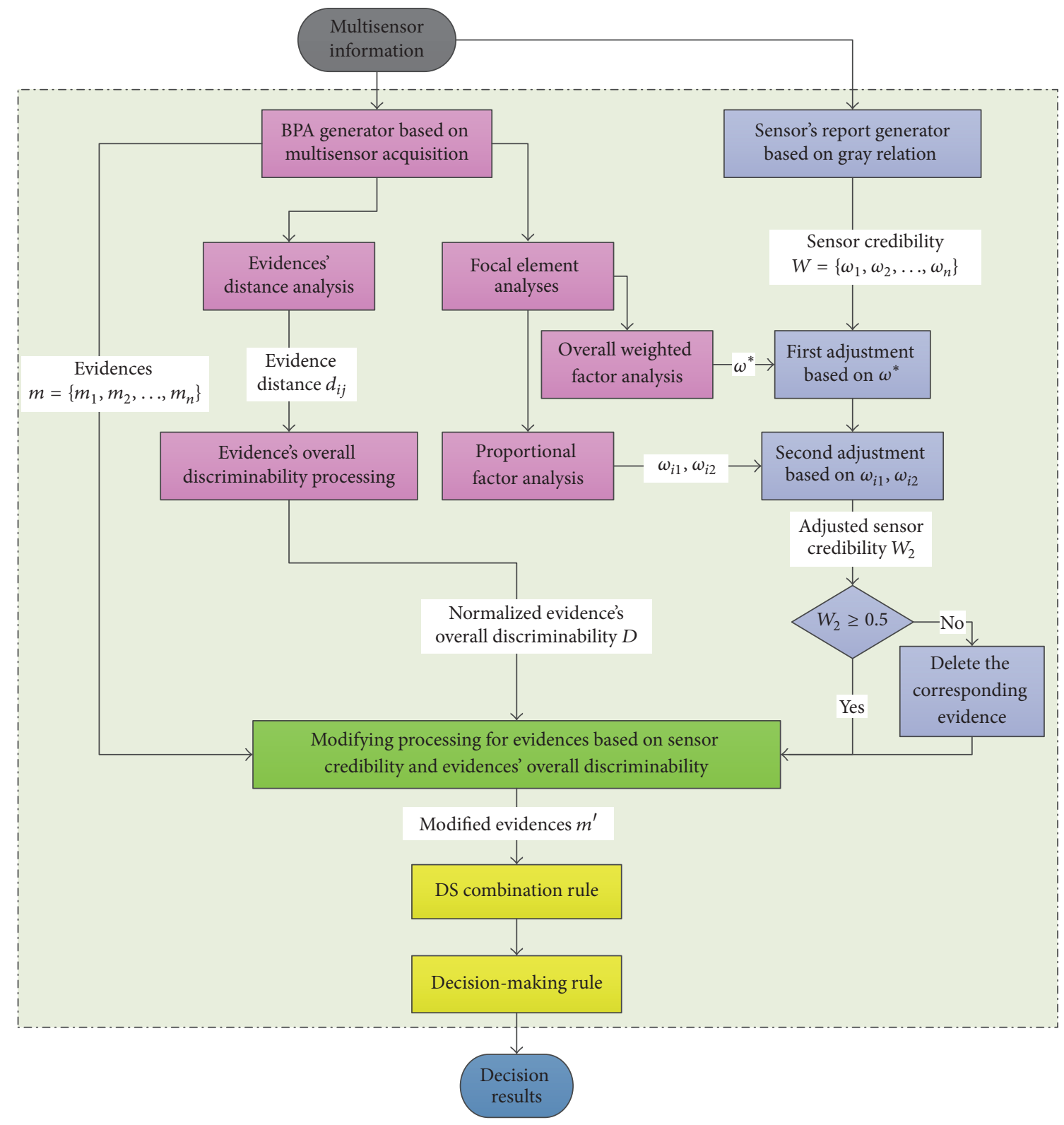

FIGURE 3: Flow chart of the novel decision-making algorithm.

is the number of targets, and $j$ indicates the characteristic information of each sensor. Under these assumptions, we can acquire sensor's credibility with following steps.

Firstly, calculate the absolute difference of attributes as

$$
\omega_{i}(j)=\left|\mathbf{X}_{0}(j)-\mathbf{X}_{i}(j)\right|,
$$

where $|\cdot|$ represents the absolute index and $\omega_{i}(j)$ indicates the absolute difference between $\mathbf{X}_{0}$ and $\mathbf{X}_{i}$ in sensor's $j$ th attribute.

Secondly, use the classic grey relation theory to calculate relation coefficient of the $i$ th sensor.

$$
\xi_{i}(j)=\frac{\min _{i} \min _{j} \omega_{i}(j)+\rho \max _{i} \max _{j} \omega_{i}(j)}{\omega_{i}(j)+\rho \max \max _{j} \omega_{i}(j)},
$$

where $\min _{i} \min _{j} \omega_{i}(j)$ is the minimum absolute difference and the $\max _{i} \max _{j} \omega_{i}(j)$ is the maximum absolute difference. And the resolution index $\rho$ is a constant as $\rho=0.5$ in this paper.

Then, obtain the grey relation factor of the $i$ th sensor with average processing.

$$
\gamma_{i}=\frac{1}{M} \sum_{j=1}^{M} \xi_{i}(j) \cdot a(j)
$$

At last, the sensor's credibility of the $i$ th sensor is shown as

$$
\omega_{i}=\frac{\gamma_{i}}{\max _{i}\left(\gamma_{i}\right)}
$$


4.1.2. Two Consecutive Adjustments of Sensor's Credibility Based on Focal Element Analysis. In order to guarantee the normalization of the synthetic results, the sum of all sensors' credibility should be unit. However, due to the influence of noise and imprecise device, the sum of sensors' credibility is not always unit. To make the final decision for information fusion obtained from such sensors, sensor's credibility and the information provided by sensors should be considered simultaneously. In this section, we discuss how to combine sensor's credibility with focal element analyses to make the final decision.

From what is mentioned above, we suppose that $\Theta=$ $\left\{m_{i}\left(H_{j}\right) \mid i=1,2, \ldots, n, j=1,2, \ldots, m\right\}$ is the FoD of system, and $m_{i}\left(H_{j}\right)$ are BPAs of focal element. $i$ is the number of sensors and $H_{j}$ represents the $j$ th focal element.

To begin with, sensors' credibility is obtained through grey relation algorithm as

$$
W=\left\{\omega_{1}, \omega_{2}, \ldots, \omega_{n}\right\}
$$

The consecutive adjustments are based on the compatibility and conflict processing of focal elements.

Primarily, the similarity and conflict between two evidences can be defined separately as

$$
\begin{aligned}
E_{i j} & =\sum_{p=q=1}^{m} m_{i}\left(F_{p}\right) \cdot m_{j}\left(F_{q}\right), \\
C_{i j} & =\sum_{p=q=1, p \neq q}^{m} m_{i}\left(F_{p}\right) \cdot m_{j}\left(F_{q}\right) .
\end{aligned}
$$

With the introduction of similarity and conflict concepts, the proportional conflict factor of the $i$ th sensor can be confirmed, which reflects the conflict level of the $i$ th evidence.

$$
k_{i}=\frac{\sum_{j=1, j \neq i}^{n} C_{i j}-\sum_{j=1, j \neq i}^{n} E_{i j}}{\sum_{j=1, j \neq i}^{n} C_{i j}+\sum_{j=1, j \neq i}^{n} E_{i j}} .
$$

Then, the average conflict coefficient $k^{*}$ of all evidences can be calculated as

$$
k^{*}=\frac{1}{2}\left(1+\frac{1}{n} \sum_{i=1}^{n} k_{i}\right) \text {. }
$$

After that, define the overall weight factor of all evidences $\omega^{*}$ according to $k^{*}$.

$$
\omega^{*}=n \cdot\left(k^{*}\right)^{\alpha} \cdot \min \left\{\omega_{i} \mid i=1,2, \ldots, n\right\},
$$

where $\alpha$ is the regulatory factor, and the related analysis is discussed in Section 5.1.

Finally, the adjustments of sensors' credibility are based on different processing of $\omega^{*}$. One is based on $\omega^{*}$ itself, and the other is based on two parts of $\omega^{*}$ as the proportion of compatible focal elements and the proportion of conflict focal elements. Thus, the first and the second adjustment for all sensors' credibility are, respectively,

$$
\begin{aligned}
W_{1} & =\left\{\omega_{1}-\frac{1}{n} \omega^{*}, \omega_{2}-\frac{1}{n} \omega^{*}, \ldots, \omega_{n}-\frac{1}{n} \omega^{*}\right\}, \\
W_{2} & =\left\{\omega_{1}-\frac{1}{n} \omega^{*}+\omega_{11}+\omega_{12}, \omega_{2}-\frac{1}{n} \omega^{*}+\omega_{21}\right. \\
& \left.+\omega_{22}, \ldots, \omega_{n}-\frac{1}{n} \omega^{*}+\omega_{n 1}+\omega_{n 2}\right\},
\end{aligned}
$$

where $\omega_{i 1}, \omega_{i 2}$ separately represent the proportion of compatible focal elements and the proportion of conflict focal elements, which are defined as

$$
\begin{aligned}
& \omega_{i 1}=\frac{E_{i}}{\sum_{i=1}^{n} E_{i}} \omega_{1}^{*}, \\
& \omega_{i 2}=\frac{1 / C_{i}}{\sum_{i=1}^{n}\left(1 / C_{i}\right)} \omega_{2}^{*} .
\end{aligned}
$$

$W_{2}$ is the modified sensors' credibility, in which the conflict among evidences can be reflected. When the sensor's credibility of certain evidence is very small, it indicates that this evidence has big conflict with all the other evidences. Thus, a threshold is indispensable for dealing with sensor's credibility which can help system to delete those evidences with low sensor's credibility. In this paper, the threshold is set to 0.5 .

4.2. Establishment of Evidence's Overall Discriminability Based on Evidences' Distance Processing. Firstly, the form of evidences' distance function is introduced, which can distinguish the evidences' difference.

$$
\begin{aligned}
d & \left(\mathbf{m}_{1}, \mathbf{m}_{2}\right) \\
& =\sqrt{\frac{1}{2}\left(\left\langle\mathbf{m}_{1}, \mathbf{m}_{1}\right\rangle+\left\langle\mathbf{m}_{2}, \mathbf{m}_{2}\right\rangle-2 \times\left\langle\mathbf{m}_{1}, \mathbf{m}_{2}\right\rangle\right)}
\end{aligned}
$$

in which

$$
\left\langle\mathbf{m}_{1}, \mathbf{m}_{2}\right\rangle=\sum_{i=1}^{2^{N}} \sum_{j=1}^{2^{N}} \mathbf{m}_{1}\left(A_{i}\right) \mathbf{m}_{2}\left(A_{j}\right) \frac{\left|A_{i} \cap A_{j}\right|}{\left|A_{i} \cup A_{j}\right|},
$$

where $|\cdot|$ indicates the number of focal elements.

According to the property that two evidences are more similar with smaller distance function, we can define evidences' overall discriminability as

$$
D_{i}=\sum_{j=1}^{m} d_{i j}
$$

And for the normalization feature of the synthetic results, $D_{i}$ should be normalized.

$$
D_{i}(\text { norm })=\frac{\left(\left(1 / D_{i}\right) / \sum_{i=1}^{m}\left(1 / D_{i}\right)\right)}{\sum\left(\left(1 / D_{i}\right) / \sum_{i=1}^{m}\left(1 / D_{i}\right)\right)} .
$$

It can be easily proved that $D_{i}$ reflects the incompatibility degree between the $i$ th evidence and all the other evidences. That is, the larger $D_{i}$ is, the less the support degree can be obtained, and the worse the evidence's credibility will be. 
4.3. Modification of Evidences. Taking sensor's credibility and evidence's overall discriminability simultaneously into consideration, the modified evidences can be expressed as

$$
\begin{aligned}
m_{i}\left(H_{j}\right)= & W_{2}(i) m_{i}\left(H_{j}\right) \\
& +e^{-k}\left(1-D_{i}\right)\left(W_{2}(i)-D_{i}\right), \\
m_{i}(\Theta)= & 1-\sum_{j=1}^{m} m_{i}\left(H_{j}\right)
\end{aligned}
$$

where $k$ is the global conflict factor.

The modification of evidences takes full advantage of sensor's credibility and real-time information provided by sensors to ameliorate evidences. If one modified evidence has zero focal element, we choose to delete the evidence and replace it with the average of other evidences. This procedure will not only guarantee a reasonable fusion results, but also effectively avoid the occurrence of one-vote veto when evidences combine.

4.4. Combination of Modified Evidences. Finally, the modified evidence is integrated with the comprehensive DS combination rule to make the final judgment.

Consider that the combination results satisfy

$$
\begin{aligned}
& m\left(H_{1}\right)=\max \left\{m\left(H_{i}\right), H_{i} \subset \Theta\right\}, \\
& m\left(H_{2}\right)=\max \left\{m\left(H_{j}\right), H_{j} \subset \Theta, H_{j} \neq H_{1}\right\}, \\
& m\left(H_{1}\right) \geq \varepsilon_{1}, \\
& m\left(H_{1}\right)-m\left(H_{2}\right) \geq \varepsilon_{2} .
\end{aligned}
$$

$H_{1}$ is the decision-making result through the novel algorithm, where $\varepsilon_{1}$ and $\varepsilon_{2}$ are preset threshold values. Otherwise, $\Theta$ is the result, which means that the system cannot be identified rationally.

\section{Simulation and Comparative Analyses}

This section is divided into two parts. One is the experiment preparation that discusses the value of the regulatory factor $\alpha$, and the other is effectiveness validation of the new decisionmaking method.

5.1. Experiment Preparation. Prior to the experiment, the analysis about the accurate expression of evidences' conflict and the selection of the regulatory factor are described in this section.

5.1.1. Precise Expression of Conflict. An experiment is carried out to prove the effectiveness of the improved algorithm in expressing evidences' conflict.

Assume that FoD is $\Theta=\{A, B, C\}$, where $A, B, C$ are mutually exclusive. The standard and reference sensor's judgment value is $m_{0}=\{0.5,0.3,0.2\}$. Ten groups of sensor's judgment values obtained by multisensor data fusion system
TABLE 1: Ten sensors' BPAs and their credibility.

\begin{tabular}{lcccc}
\hline \multirow{2}{*}{ Sensors } & \multirow{2}{*}{ Sensor's credibility } & \multicolumn{3}{c}{ Propositions } \\
& & $A$ & $B$ & $C$ \\
\hline Sensor 1 & 0.7094 & 0.5853 & 0.3791 & 0.0357 \\
Sensor 2 & 0.5777 & 0.1680 & 0.5756 & 0.2565 \\
Sensor 3 & 0.6266 & 0.2591 & 0.3936 & 0.3473 \\
Sensor 4 & 0.5781 & 0.3938 & 0.5982 & 0.0080 \\
Sensor 5 & 0.6304 & 0.7387 & 0.1461 & 0.1151 \\
Sensor 6 & 0.8882 & 0.5560 & 0.2960 & 0.1480 \\
Sensor 7 & 0.5953 & 0.2870 & 0.5881 & 0.1249 \\
Sensor 8 & 0.4556 & 0.0120 & 0.5957 & 0.3923 \\
Sensor 9 & 0.9040 & 0.5462 & 0.2728 & 0.1810 \\
Sensor 10 & 0.6018 & 0.2893 & 0.5814 & 0.1293 \\
\hline
\end{tabular}

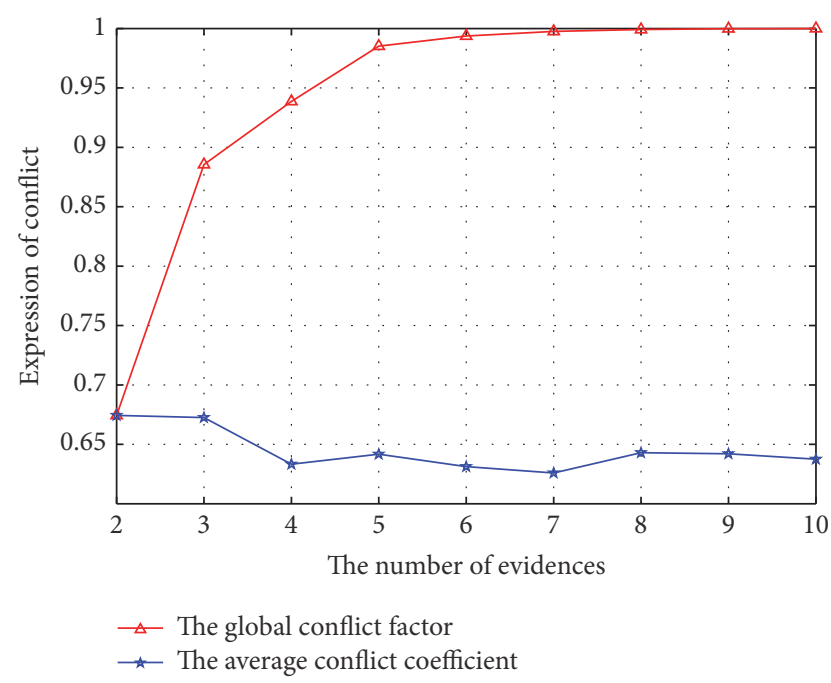

FIgURE 4: Comparison between the global conflict factor and the average conflict coefficient.

and the corresponding sensor's credibility are shown in Table 1 .

According to Table 1, the comparison between the global conflict factor $k$ in DS evidence theory and the average conflict coefficient $k^{*}$ in the novel method is shown in Figure 4.

It is obvious in Figure 4 that $k$ in DS evidence theory is getting larger along with the increasing of evidences' number. However, the acquisition of evidences is the processing to get support for propositions, not the processing to get more conflict. Thus, $k$ is not able to accurately represent the conflict situation. However, $k^{*}$ in the novel method is the effective expression of actual evidences' conflict. Thus, Figure 4 indirectly illustrates the rationality of the new decision-making method.

5.1.2. Analysis of the Regulatory Factor. During the consecutive adjustments of sensor's credibility, there is an indispensable index as the regulatory factor $\alpha$. To analyze the numerical selection of $\alpha$, statistical methods are adopted. As the modified sensor's credibility $W_{2}$ is partially determined by 


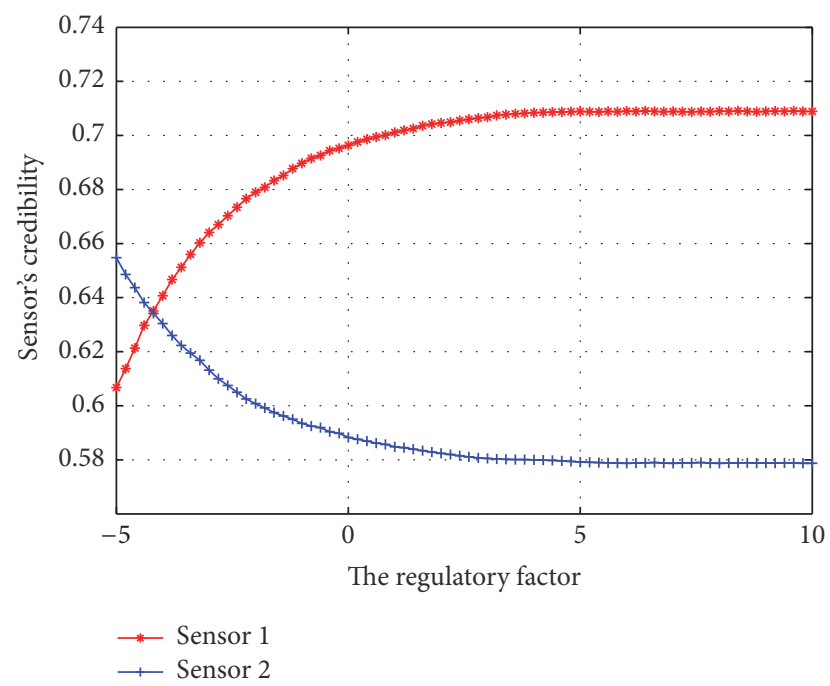

FIGURE 5: Relationship of sensor's credibility and the regulatory factor with two evidences.

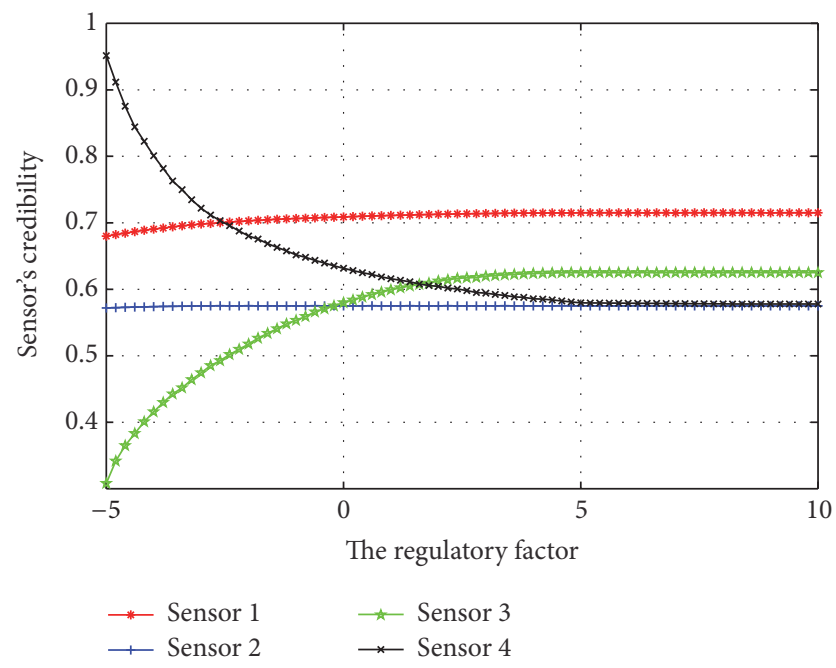

FIGURE 6: Relationship of sensor's credibility and the regulatory factor with four evidences.

$\alpha$, the relationship between the regulatory factor and sensor's credibility with 2 evidences is indicated in Figure 5.

As can be seen from Figure 5, with the increasing of the regulatory factor $\alpha$, sensor's credibility gradually tends to be stable. It proves that the perfect regulatory factor can be confirmed.

In order to further reflect the numerical range of $\alpha$, the number of sensors is increased to finish the simulation. Figure 6 shows the relationship of sensor's credibility and the regulatory factor with 4 evidences.

From Figure 6, it is clear that sensor's credibility tends to be relatively stable when the regulatory factor reaches 5 . Thus, the regulatory factor value is set to 5 in the next experiment.

5.2. Effectiveness Validation of the New Decision-Making Method. In this experiment, the proposed algorithm is
TABLE 2: Four sensors' BPAs and their credibility.

\begin{tabular}{lcccc}
\hline \multirow{2}{*}{ Sensors } & \multirow{2}{*}{ Sensor's credibility } & \multicolumn{3}{c}{ Propositions } \\
& & $A$ & $B$ & $C$ \\
\hline Sensor 1 & 0.7563 & 0.5853 & 0.3791 & 0.0357 \\
Sensor 2 & 0.6182 & 0.3938 & 0.5982 & 0.0080 \\
Sensor 3 & 0.4792 & 0.0000 & 0.5756 & 0.4244 \\
Sensor 4 & 0.9595 & 0.5462 & 0.2728 & 0.1810 \\
\hline
\end{tabular}

TABLE 3: The fusion result of 2 sensors.

\begin{tabular}{lcccc}
\hline \multirow{2}{*}{ Algorithms } & \multicolumn{4}{c}{ Propositions } \\
& $A$ & $B$ & $C$ & $\Theta$ \\
\hline LIU & 0.5004 & 0.4986 & 0.0010 & 0.0000 \\
YAGER & 0.5037 & 0.4956 & 0.0006 & 0.0000 \\
GUO & 0.4972 & 0.5000 & 0.0028 & 0.0000 \\
LI & 0.5041 & 0.4727 & 0.0001 & 0.0231 \\
TAN & 0.4794 & 0.4653 & 0.0008 & 0.0546 \\
CHENG & 0.5018 & 0.4982 & 0.0000 & 0.5018 \\
CHEN & 0.5018 & 0.4982 & 0.0000 & 0.5018 \\
HE & 0.3258 & 0.3206 & 0.0004 & 0.3532 \\
YE & 0.3864 & 0.2652 & 0.2955 & 0.0530 \\
YAO & 0.4960 & 0.4918 & 0.0121 & 0.0000 \\
FLOREA & 0.3263 & 0.3257 & 0.0146 & 0.3334 \\
MURPHY & 0.5004 & 0.4986 & 0.0010 & 0.0000 \\
Proposed method & 0.5079 & 0.4786 & 0.0134 & 0.0000 \\
\hline
\end{tabular}

compared with other methods to prove its priority in overcoming problems such as high conflict and one-vote veto and ulteriorly realizing uncertain data fusion correctly.

Assume that $\mathrm{FoD}$ is $\Theta=\{A, B, C\}$, where $A, B, C$ are mutually exclusive. The standard and reference sensor's judgment value is $m_{0}=\{0.5,0.3,0.2\}$.

Four groups of sensor's judgment values obtained by multisensor data fusion system and the corresponding sensor's credibility are shown in Table 2.

It is checked in Table 2 that the commonsensical fusion result should give proposition $A$ the largest support as two sensors with big credibility both support proposition $A$ to a great extent. With similar principle, proposition $C$ in fusion result should own the minimum support.

The data fusion of 4 sensors is divided into 3 steps. And we take 12 common improved methods in [20-31] as the compared algorithms. These methods are separately abbreviated as LIU [20], YAGER [21], GUO [22], LI [23], TAN [24], CHENG [25], CHEN [26], HE [27], YE [28], YAO [29], FLOREA [30], and MURPHY [31].

Firstly, the data fusion of sensor 1 and sensor 2 is achieved and the result is shown in Table 3.

From Table 3, we can see that all methods give proposition $A$ the largest support except GUO, which demonstrates that GUO makes the wrong decision. Moreover, CHENG, CHEN, and FLOREA allocate $\Theta$ a lot of support, which is not conducive to final judgment. Concerning method YE, the fusion result is averagely allocated to each proposition, in which the support to proposition $C$ mismatches with the 
TABLE 4: The fusion result of 3 sensors.

\begin{tabular}{lcccc}
\hline \multirow{2}{*}{ Algorithms } & \multicolumn{4}{c}{ Propositions } \\
& $A$ & $B$ & $C$ & $\Theta$ \\
\hline LIU & 0.1660 & 0.8299 & 0.0041 & 0.0000 \\
YAGER & 0.0000 & 0.9991 & 0.0009 & 0.0000 \\
GUO & 0.3813 & 0.4548 & 0.1639 & 0.0000 \\
LI & 0.0000 & 0.7451 & 0.0000 & 0.2549 \\
TAN & 0.0000 & 0.6707 & 0.0008 & 0.3285 \\
CHENG & 0.2623 & 0.7301 & 0.0076 & 0.0000 \\
CHEN & 0.0000 & 1.0000 & 0.0000 & 0.0000 \\
HE & 0.0000 & 0.5756 & 0.4244 & 0.0000 \\
YE & 0.2864 & 0.3652 & 0.2955 & 0.0530 \\
YAO & 0.3282 & 0.5856 & 0.0862 & 0.0000 \\
FLOREA & 0.1399 & 0.2218 & 0.0669 & 0.5714 \\
MURPHY & 0.2671 & 0.6719 & 0.0610 & 0.0000 \\
Proposed method & 0.5193 & 0.4797 & 0.0010 & 0.0000 \\
\hline
\end{tabular}

supporting degree proved by original evidences. Although LIU, YAGER, LI, TAN, HE, YAO, and MURPHY offer proposition A the largest support, the numerical difference of support to propositions $A$ and $B$ is too tiny to facilitate decision-making fusion. Thus, only the improved method can get the proper fusion result.

In addition, sensor 3 is added in uncertain data fusion to strengthen effectiveness validation of the new decisionmaking method. Table 4 is the fusion result of 3 sensors.

As can be seen from Table 2, sensor 3 is significantly different from others which leads to high conflict, and the support to proposition $A$ is zero which leads to zero focal element. In view of the particularity properties of sensor 3 , we can see in Table 4 that one-vote veto phenomenon exists in YAGER, LI, TAN, CHEN, and HE. It reveals that the appearance of zero focal element directly deteriorates the fusion result. FLOREA still assigns a lot of support to $\Theta$ and increases the uncertainty in fusion result. LIU, GUO, CHENG, YE, FLOREA, and MURPHY are unable to reasonably handle zero focal element and utilize sensor's credibility. The fusion results of them all give proposition $B$ the excessive support as the incorporation of sensor 3 . The proposed algorithm modifies the 3rd evidence via taking sensor's credibility into account as well as the overall situation of all evidence's discriminability, which reduces its influence on fusion result. Thus, in the fusion of 3 sensors, the proposed method is still the optimal resolution for uncertain data fusion.

Finally, in order to verify the priority of the proposed method, evidence with relatively higher sensor credibility is imported, and the data fusion is accomplished with 4 sensors. The decision-making processing is also completed, whose result is displayed in Table 5. The threshold values in decisionmaking rule are $\varepsilon_{1}=0.40$ and $\varepsilon_{2}=0.15$.

We can see from Table 5 that the occurrence of zero focal element in sensor 3 seriously affects the data fusion. Even sensor 4 with large sensor's credibility supports proposition $A$ explicitly, one-vote veto phenomenon still exists in YAGER, LI, TAN, CHEN, and HE, and the decision fusions of LIU and
TABLE 5: The decision result of 4 sensors.

\begin{tabular}{lccccc}
\hline \multirow{2}{*}{ Algorithms } & \multicolumn{5}{c}{ Propositions } \\
& $A$ & $B$ & $C$ & $\Theta$ & Decision result \\
\hline LIU & 0.3136 & 0.6863 & 0.0001 & 0.0000 & $B$ \\
YAGER & 0.0000 & 0.9994 & 0.0006 & 0.0000 & $B$ \\
GUO & 0.4414 & 0.4055 & 0.1531 & 0.4414 & $\Theta$ \\
LI & 0.0000 & 0.7506 & 0.0000 & 0.2494 & $B$ \\
TAN & 0.0000 & 0.8664 & 0.0004 & 0.1332 & $B$ \\
CHENG & 0.4520 & 0.5401 & 0.0079 & 0.4520 & $\Theta$ \\
CHEN & 0.0000 & 1.0000 & 0.0000 & 0.0000 & $B$ \\
HE & 0.0000 & 0.1013 & 0.0001 & 0.8986 & $\Theta$ \\
YE & 0.5462 & 0.2728 & 0.1810 & 0.0000 & $A$ \\
YAO & 0.4339 & 0.4617 & 0.1045 & 0.4339 & $\Theta$ \\
FLOREA & 0.1173 & 0.1404 & 0.0499 & 0.6923 & $\Theta$ \\
MURPHY & 0.3826 & 0.5481 & 0.0693 & 0.0000 & $B$ \\
Proposed method 0.7137 & 0.2860 & 0.0003 & 0.0000 & $A$ \\
\hline
\end{tabular}

MURPHY give the wrong decision results to proposition $B$, while the decision fusion of FLOREA sequentially regards $\Theta$ as the decision result. Secondly, due to the preset of threshold values in decision-making rule, GUO, CHENG, and YAO consider $\Theta$ as the decision result. Moreover, only YE and the proposed method generate reasonable decision results as they take proposition $A$ as the final decision. Compared with YE, the proposed method assigns larger support to proposition $A$, which is beneficial to get the precise decision result. Thus, the proposed method is more rational and reliable.

The data fusion of 4 sensors above reflects that the proposed method makes the reliable and accurate decision in comprehensive consideration of sensor's credibility and overall evidence's discriminability. Besides, the decision result reveals that the proposed method will not only give accurate decision, but also avoid harmful effects caused by sensors with low credibility and zero focal elements.

\section{Conclusion}

As multisensor information fusion is broadly applied in many civil and military areas, the valid decision-making method for uncertain information fusion is under great attention. This paper raises a neoteric decision-making algorithm based on grey relation and DS evidence theory to solve the uncertainty caused by inconsistence of sensors itself and complexity of monitoring environment. The new algorithm is carried out with three innovative treatments: generation of sensor's credibility based on grey relation theory, focal element analyses as overall weighted factor analysis and proportional factor analysis, and evidences' overall discriminability processing. Simulation results and analyses show that the proposed algorithm can make precise decision without worrying about sensors' unreliability and evidence's high conflict. Thus, it has great application significance and excellent engineering prospect.

In further study, the decision-making method for uncertain data fusion should pay close attention to relieve the huge computation burden for system as the increasing number of 
sensors and try to realize the on-time and on-line decisionmaking system.

\section{Competing Interests}

The authors declare that there is no conflict of interests regarding the publication of this paper.

\section{Acknowledgments}

The paper is funded by the National Key Research and Development Program of China (Grant no. 2016YFF0102806), the National Natural Science Foundation of China (Grant no. 51509049), the Natural Science Foundation of Heilongjiang Province, China (Grant no. F201345), and the Fundamental Research Funds for the Central Universities of China (no. GK2080260140).

\section{References}

[1] J. A. Benediktsson and I. Kanellopoulos, "Classification of multisource and hyperspectral data based on decision fusion," IEEE Transactions on Geoscience and Remote Sensing, vol. 37, no. 3, pp. 1367-1377, 1999.

[2] M. Daniel, "Distribution of contradictive belief masses in combination of belief functions," in Information, Uncertainty and Fusion, pp. 431-446, Springer, Berlin, Germany, 2000.

[3] L. Dymova and P. Sevastjanov, "An interpretation of intuitionistic fuzzy sets in terms of evidence theory: decision making aspect," Knowledge-Based Systems, vol. 23, no. 8, pp. 772-782, 2010.

[4] P. A. Samara, G. N. Fouskitakis, J. S. Sakallariou, and S. D. Fassois, "A statistical method for the detection of sensor abrupt faults in aircraft control systems," IEEE Transactions on Control Systems Technology, vol. 16, no. 4, pp. 789-798, 2008.

[5] X. L. Zhu, Fundamentals of Applied Information Theory, Tsinghua University Press, Beijing, China, 2001.

[6] M. Truchon, "Borda and the maximum likelihood approach to vote aggregation," Mathematical Social Sciences, vol. 55, no. 1, pp. 96-102, 2008.

[7] Z.-J. Zhou, C.-H. Hu, D.-L. Xu, J.-B. Yang, and D.-H. Zhou, "Bayesian reasoning approach based recursive algorithm for online updating belief rule based expert system of pipeline leak detection," Expert Systems with Applications, vol. 38, no. 4, pp. 3937-3943, 2011.

[8] S.-H. Oh, "Improving the error backpropagation algorithm with a modified error function," IEEE Transactions on Neural Networks, vol. 8, no. 3, pp. 799-803, 1997.

[9] Y. Deng, “Generalized evidence theory," Applied Intelligence, vol. 43, no. 3, pp. 530-543, 2015.

[10] H. Li, G. Wen, Z. Yu, and T. Zhou, "Random subspace evidence classifier," Neurocomputing, vol. 110, pp. 62-69, 2013.

[11] Z. He, H. Zhang, J. Zhao, and Q. Qian, "Classification of power quality disturbances using quantum neural network and DS evidence fusion," European Transactions on Electrical Power, vol. 22, no. 4, pp. 533-547, 2012.

[12] G. Dong and G. Kuang, "Target recognition via information aggregation through Dempster-Shafer's evidence theory," IEEE Geoscience and Remote Sensing Letters, vol. 12, no. 6, pp. 12471251, 2015.
[13] F. Ye, Y. Li, R. Yang, and Z. Sun, “The user requirement based competitive price model for spectrum sharing in cognitive radio networks," International Journal of Distributed Sensor Networks, vol. 9, no. 11, Article ID 724581, 2013.

[14] X. Fan and M. J. Zuo, "Fault diagnosis of machines based on D-S evidence theory-part 1: D-S evidence theory and its improvement," Pattern Recognition Letters, vol. 27, no. 5, pp. 366-376, 2006.

[15] J.-C. Li, Y.-B. Li, S. Kidera, and T. Kirimoto, "A robust signal recognition method for communication system under timevarying SNR environment," IEICE Transactions on Information and Systems, vol. E96-D, no. 12, pp. 2814-2819, 2013.

[16] M. Beynon, D. Cosker, and D. Marshall, "An expert system for multi-criteria decision making using Dempster-Shafer theory," Expert Systems with Applications, vol. 20, no. 4, pp. 357-367, 2001.

[17] Y. Deng, "Deng entropy," Chaos, Solitons and Fractals, vol. 91, pp. 549-553, 2016.

[18] W. Jiang, B. Wei, C. Xie et al., "An evidential sensor fusion method in fault diagnosis," Advances in Mechanical Engineering, vol. 8, no. 3, pp. 1-7, 2016.

[19] A.-L. Jousselme, D. Grenier, and É. Bossé, "A new distance between two bodies of evidence," Information Fusion, vol. 2, no. 2, pp. 91-101, 2001.

[20] Y.-Z. Liu, Y.-C. Jiang, and J.-K. Zhang, "Utility analysis of belief in evidence theory," System Engineering Theory and Practice, vol. 28, no. 3, pp. 103-110, 2008.

[21] R. R. Yager, "On the dempster-shafer framework and new combination rules," Information Sciences, vol. 41, no. 2, pp. $93-$ 137, 1987.

[22] H. Guo, W. Shi, Q. Liu et al., "A new combination rule of evidence," Journal of Shanghai Jiao-Tong University-Chinese Edition, vol. 40, no. 11, pp. 1895-1900, 2006.

[23] L. Li, D. Ma, C. Wang et al., "New method for conflict evidence processing in DS theory," Application Research of Computers, vol. 28, no. 12, pp. 4528-4531, 2011.

[24] Q. Tan and Y.-H. Xiang, "Application of weighted evidential theory and its information fusion method in fault diagnosis," Journal of Vibration and Shock, vol. 27, no. 4, pp. 112-116, 2008.

[25] H. Cheng, S.-W. Du, C.-H. Xu, and J.-J. Lin, "A DS-based multiindex fusion of information fusion algorithm," Journal of East China University of Science and Technology, vol. 37, no. 4, pp. 483-486, 2011.

[26] B. Chen and S. H. Wan, "Study on ship detection with improved Dempster-Shafer theory," Computer Engineering and Applications, vol. 46, no. 28, pp. 222-224, 2010.

[27] B. He and H.-L. Hu, "Modified DS evidence combination strategy," Acta Aeronautica et Astronautica Sinica, vol. 24, no. 6, pp. 559-562, 2003.

[28] Q. Ye, X.-P. Wu, and D.-J. Zhai, "Combination algorithm for evidence theory utilizing energy function," Systems Engineering and Electronics, vol. 32, no. 3, pp. 566-569, 2010.

[29] J. Yao, C. Wu, X. Xie, K. Qian, G. Ji, and P. Bhattacharya, "A new method of information decision-making based on D-S evidence theory," in Proceedings of the IEEE International Conference on Systems, Man and Cybernetics (SMC '10), pp. 1804-1811, Istanbul, Turkey, October 2010.

[30] M. C. Florea, A.-L. Jousselme, É. Bossé, and D. Grenier, “Robust combination rules for evidence theory," Information Fusion, vol. 10, no. 2, pp. 183-197, 2009. 
[31] C. K. Murphy, "Combining belief functions when evidence conflicts," Decision Support Systems, vol. 29, no. 1, pp. 1-9, 2000.

[32] Q. Zhang, Y. F. Tian, and Y. Liu, "Grey-relation based approach to uncertain multiple attribute decision making," in Proceedings of the IEEE International Conference on Computational Intelligence and Natural Computing (CINC '09), vol. 2, pp. 456-458, IEEE, Wuhan, China, June 2009.

[33] X. Xia, F. Meng, and T. Lv, "Grey relation method for calculation of embedding dimension and delay time in phase space reconstruction," Journal of Grey System, vol. 22, no. 2, pp. 105116, 2010.

[34] Y. Li, C. Shao, and X. Hou, "A novel grey relation analysis algorithm: uniform incidence degree," Information and ControlShenyang, vol. 35, no. 4, p. 462, 2006.

[35] J. L. Deng, The Basis of Grey Theory, Press of Huazhong University of Science and Technology, Wuhan, China, 2002. 


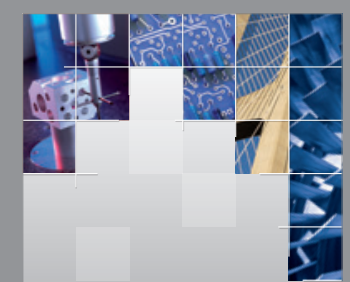

\section{Enfincering}
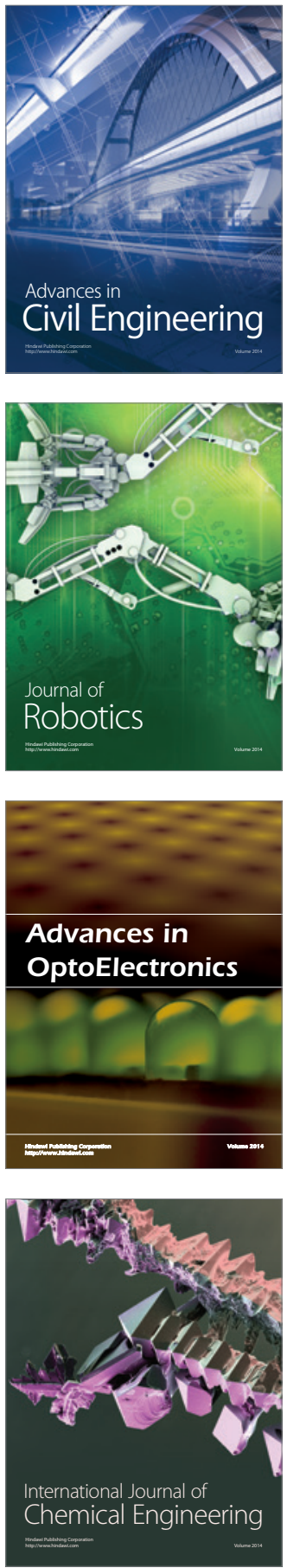

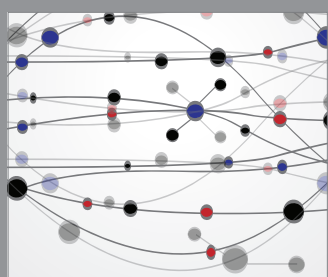

The Scientific World Journal

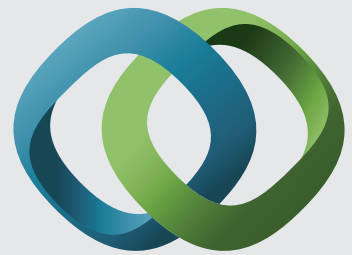

\section{Hindawi}

Submit your manuscripts at

http://www.hindawi.com
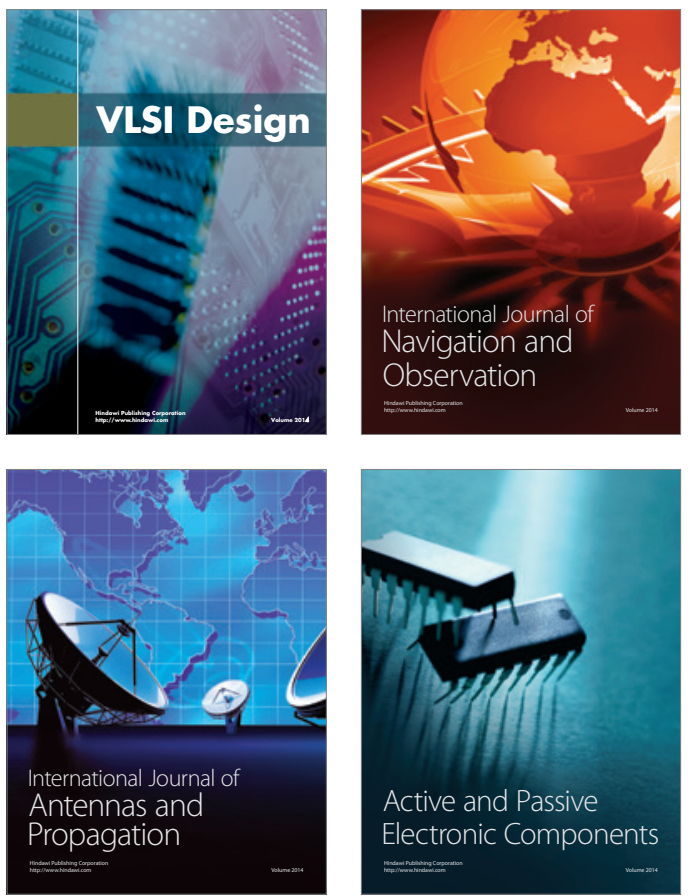
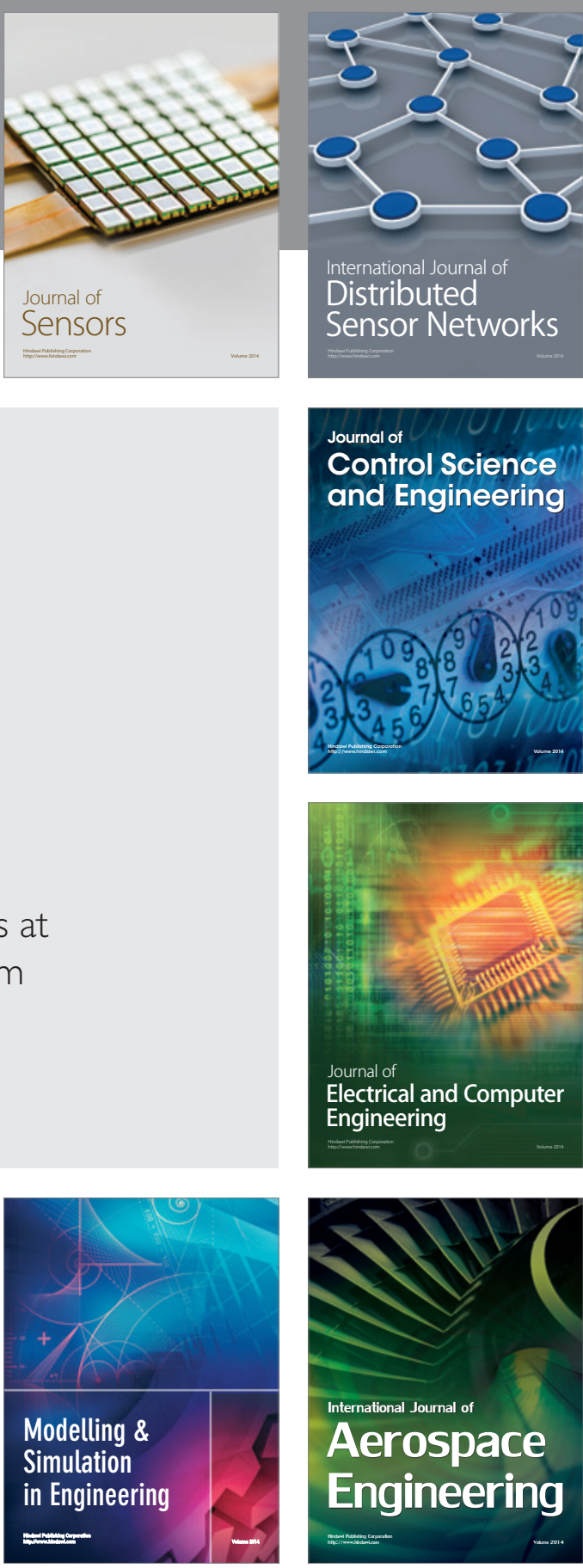

International Journal of

Distributed

Sensor Networks

Journal of

Control Science

and Engineering
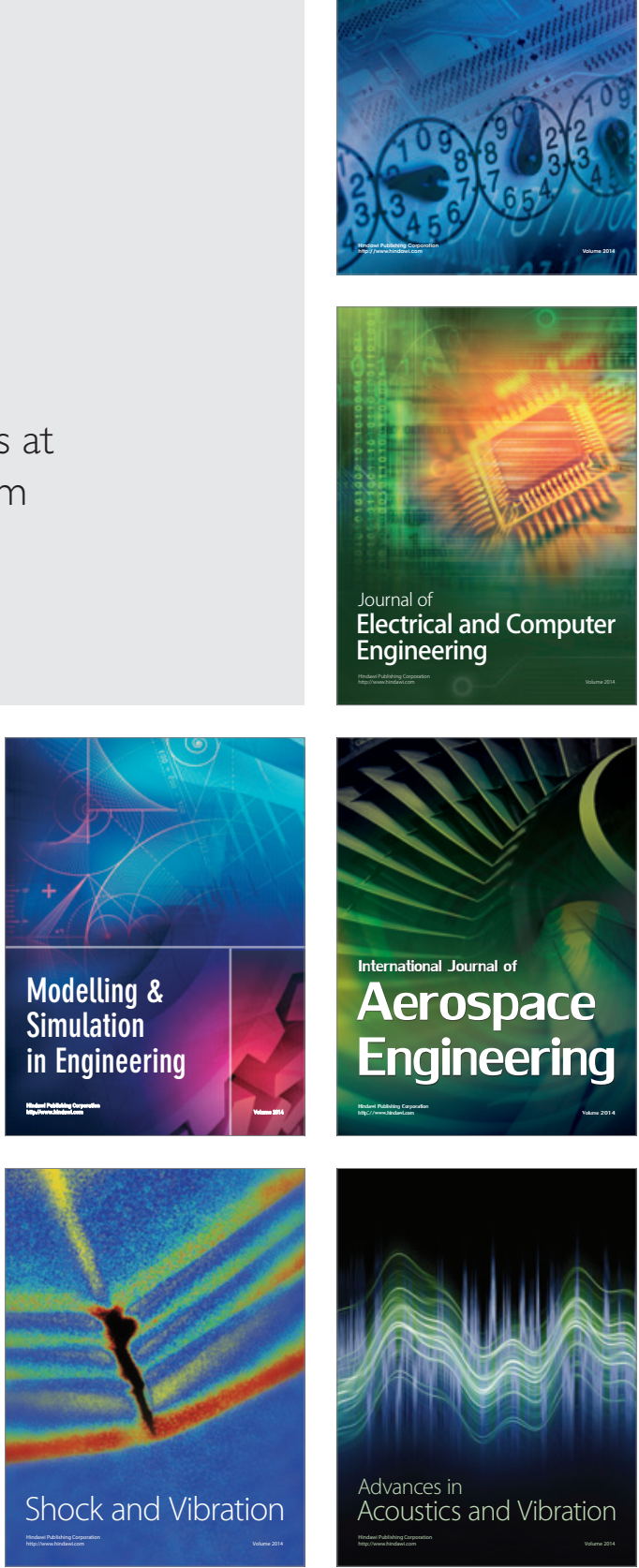